\title{
The frequency of the pathogenic bacteria isolated from different clinical samples in Duhok, Iraq
}

\author{
Fawwaz F. Ali ${ }^{1}$, Muslim A. Allu ${ }^{2}$, Mahde S. Assafi ${ }^{3}$, Mohammad I. Al-Berfkani ${ }^{2}$ \\ \{fawwaz@ntu.edu.iq ${ }^{1}$, muslimbabawat066@gmail.com², mahde.assafi@uod.ac ${ }^{3}$ \} \\ Department of Animal Production, Northern Technical University, Mosul, Iraq ${ }^{1}$, Department of Medical \\ Laboratory Technology, Duhok Polytechnic University, Duhok, Iraq ${ }^{2}$, Department of Biology, School of \\ Sciences, University of Duhok, Duhok, Iraq ${ }^{3}$
}

\begin{abstract}
Pathogenic bacteria are still considered the most global problematic issue, and they have a significant impact on human health, particularly the Enterobacteriaceae bacteria. This study aimed to investigate the frequency of the pathogenic bacteria and the related risk factors among patients in Duhok, Iraq. A total of 700 samples (281 from males and 419 from females) from Duhok Teaching Hospital were recruited and analysed. All samples were routinely cultured and identified based on their cultural and biochemical characteristics. Furthermore, the diagnosis was confirmed by using the Vitek 2 system. The results showed that the highest rate was for Staphylococcus aureus (31\%), and the lowest rate was for Candida albicans $(4.4 \%)$. The other infections varied in their rates and included $27.7 \%$ E. coli, 9.4\% Klebsiella pneumonia, 6.1\% Acinetobacter pneumonia, $6 \%$ Streptococcus spp., 5.4\% Enterobacter spp., 5.1\% Pseudomonas aeruginosa and $4.7 \%$ Proteus mirabilis. Furthermore, the bacteria were mostly recovered from urine $(67.1 \%)$ and swab (19.6\%) specimens. The infection rate was higher in females $(58.3 \%)$ than males $(41.7 \%)$. This study suggested that the incidence of pathogenic bacteria is high in Duhok, Iraq. However, it was comparable to the global bacterial pattern with the domination of $S$. aureus and E. coli.
\end{abstract}

Keywords: Pathogenic bacteria, Risk factor, S. aureus, E. coli, Duhok.

\section{Introduction}

Epidemiological studies screen diseases to determine the change of health in humans and animal populations. These studies mainly include collecting the data and transmitting this data to process and distribution [1]. Regarding bacterial infections, gram-negative bacteria are the most global problematic issue because of their high resistance to antimicrobial treatment. These bacteria have a significant impact on human health and are linked with high morbidity and case fatality within infected patients [2]. Infectious disease is the leading cause of death worldwide, and the diagnosis of infections is increasingly challenging in the clinical setting. Enterobacteriaceae present the most significant bacterial population that is clinically isolated among other gram-negative bacteria such as Acinetobacter and Pseudomonas [3]. Within the family of Enterobacteriaceae, Enterobacter spp. have been known as an important organism as these bacteria have increasingly resisted antibiotic treatment [4, 5] and commonly cause hospital-acquired pneumonia [6]. Escherichia coli (E. coli) that belongs to the Enterobacteriaceae family exposes a significant global public health problem, and it is a widely investigated bacteria and linked with many infections with a high ability to resist antimicrobial treatment [7]. P. aeruginosa is shown to be connected with high morbidity and patient deaths. Also, these bacteria have the massive ability to resist different anti-microbial treatments and are 
the most common bacteria among patients [8, 9]. Staphylococcus aureus, in particular, the methicillin-resistant Staphylococcus aureus (MRSA) strain was found to be responsible for many human and animal diseases $[10,11]$ as well as Streptococcus pneumonia that causes pneumonia, bacteremia, meningitis, otitis media, and sepsis [12]. Identifying the distribution and the risk factors of pathogens in every geographic locale is very important to understand the nature of the disease and to investigate the effective eradication program of the pathogens in humans. Studies showed that environmental factors such as geographic location, seasonal changes, and host played a role in the distribution of the pathogens [1,2]. Additionally, data showed significant seasonal differences in bacterial species worldwide [3]. In Duhok, Iraq, little data is available regarding the distribution and nature of pathogenic bacteria. Thus, this study aimed to study the epidemiology of pathogenic bacteria among patient populations in Duhok, Iraq, and to investigate the significant effects of some factors on the distribution of these pathogens.

\section{Materials and Methods}

The current study was carried out at Duhok Teaching Hospital, Dohok, Iraq. A total of 700 positive infected cases were analysed, 201 males and 419 females (the infected cases were identified by a specialist consultant and were sent to the laboratory for diagnosis to confirm the suspected disease). All collected samples were routinely cultured using blood and MacConkey agar, then incubated under the aerobic condition at $37 \mathrm{C}$ for 24 hours or for seven days (when found sterile upon the second culture). The cultures were declared contrary after seven days of incubation. Isolation and identification of the isolates were performed following their cultural characteristics, morphology, bacterial stain (Gram's, Zeihl stain, and sputum stain) and biochemical properties. The identification was confirmed by using the Vitek2 system (bioMerieux, France) [13, 14].

\subsection{Statistical analysis}

GraphPad prism 8. and SPSS software was utilized for statistical analysis.

\section{$3 \quad$ Results}

Different pathogenic isolates were detected, isolated, and identified. Among 700 patients in this study, the pathogenic isolates were gram-positive and gram-negative in addition to Candida albicans as fallow: Enterobacter spp. 38 cases, 5.4\%, Acinetobacter pneumonia 43 cases, $6.1 \%$. E. coli 194 cases, 27.7\%, Staphylococcus aureus 217 cases, 31\%, Klebsiella pneumonia 66 cases, 9.4\%, Streptococcus spp. Forty-two cases, 6\%, Pseudomonas aeruginosa 36 cases, 5.1\%, Proteus mirabilis 33 cases, $4.7 \%$, and Candida albicans 31 cases, $4.4 \%$ (Figure 1). The highest frequency of isolated bacteria from infected patients was $S$. aureus and E. coli, and they presented more than half of the total isolated pathogen. 


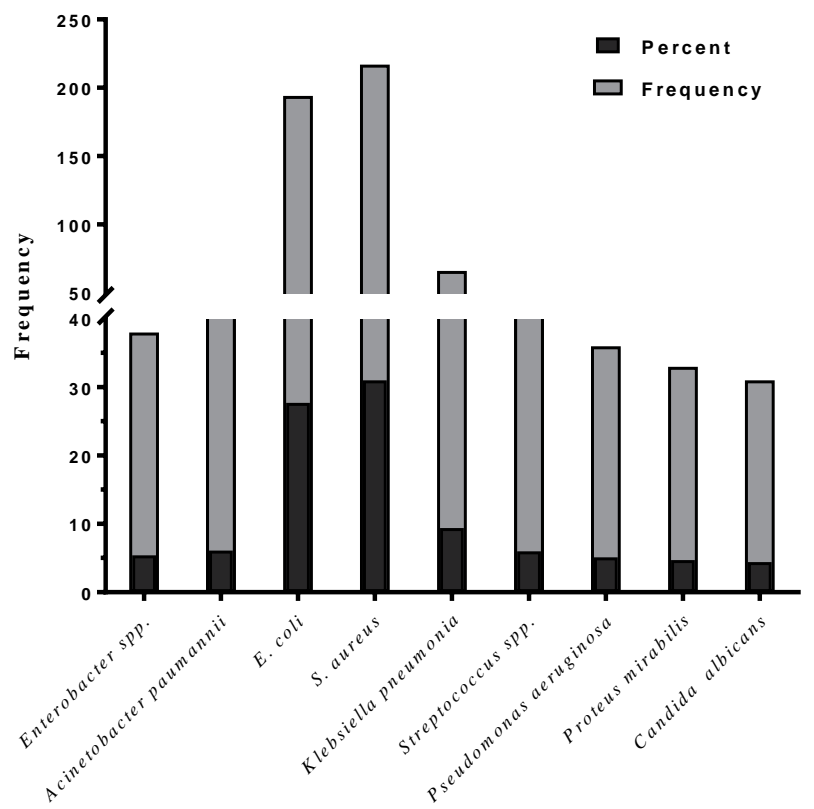

Figure 1. Frequency of isolates pathogens. These pathogens were isolated from patients that were referred to the hospital lab to confirm the clinical diagnosis. The samples were collected under the sterile condition and plated using suitable media, then identified (as mentioned in materials and methods). The data was analysed using SPSS and GraphPad prism 8.

This study also demonstrated the type and site of samples collected in which the bacteria were isolated. The data showed the bacteria were mostly recovered from urine and swab specimens (67.1\% and $19.6 \%$ respectively), (Figure 2). Furthermore, the percentage of isolated bacteria was highest in females compared with males (58.3 and 41.7, respectively) (Figure 3). 


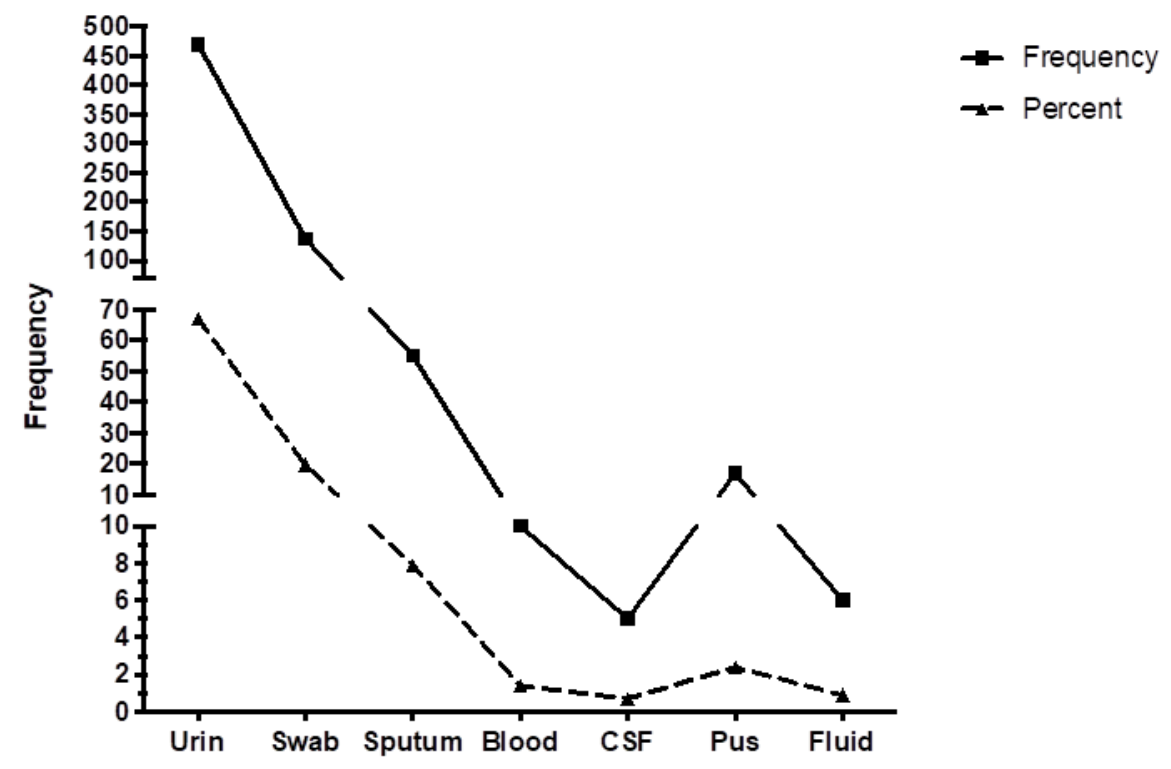

Figure 2. Frequency of pathogens distributed according to the collected specimens. Samples were collected from different sites for each patient to identify the localisation of infected pathogens. The sterile condition was involved in all collected samples and methods. The data were analysed using SPSS and GraphPad prism 8.

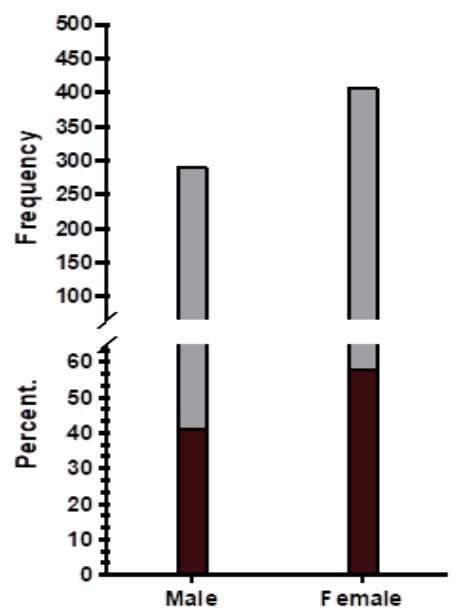

Percent

$\square$ Frequency

Figure 3: Frequency of confirmed isolates distributed according to gender. The total confirmed isolates distributed depending on the gender of patients admitted to the hospital. The data was analysed using SPSS and GraphPad prism 8. 
The results of the frequency of isolated bacteria distributed among the time of year showed a noticeable increase in the rate of isolates in February and August and October $(9.9 \%, 11.6 \%$, and $11.1 \%$, respectively).

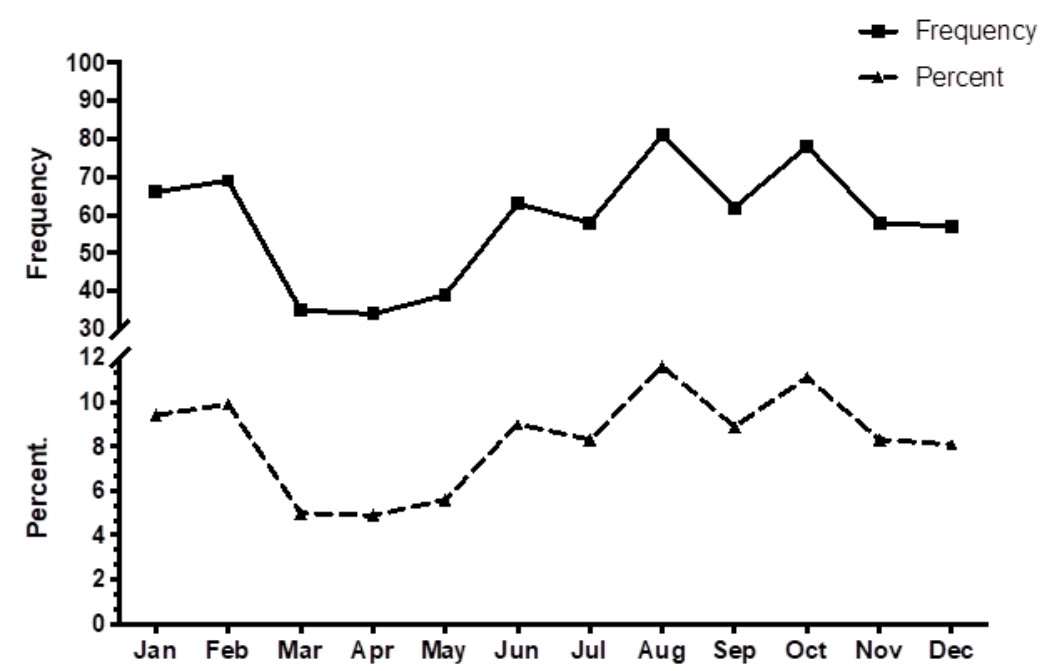

Figure 4. Frequency of confirmed isolates distributed according to the time of the year. The data were analysed using SPSS and GraphPad prism 8.

\section{Discussion}

Disease screening has been increasingly important to study disease distribution and support disease control in both humans and animals [4]. Bacterial infections are widely distributed and pose a human threat [5]. The most significant percentage of isolated bacteria from clinical samples / Dohok hospital was S. aureus, with $31 \%$ of total isolated pathogens. It seems to be the main isolated Gram-positive bacteria that cause human infections and is responsible for many human and animal diseases, in particular, the methicillin-resistant strain (MRSA) [6-8]. A study carried out at the same province to estimate the colonisation of $S$. aureus, and $18.4 \%$ of S. aureus showed to be colonised among secondary school students on Duhok city, and $2 \%$ of isolated bacteria showed resistance to Methicillin (MRSA strain) [9]. However, the prevalence of MRSA strain was revealed to be higher in healthcare workers in the same region [10]. As the results showed that $S$. aureus had the highest occurrence among other bacterial isolates, and further study might be needed to isolate the MRSA strains within these isolated bacteria.

Regarding S. pyogenes, about $92 \%$ was recovered from blood, while about $8 \%$ were isolated from CSF, peritoneal fluid, synovial fluid, and pleural fluid [11]. Although S. pyogenes can be isolated from the bloodstream in most patients, the mechanisms used by invasive strains of $S$. pyogenes to disseminate from the primary site of infection are still unclear [12]. Another grampositive bacteria, Streptococcus pneumonia, showed about $6 \%$ of isolated bacteria. This pathogen is responsible for many types of infections, such as pneumonia, bacteremia, meningitis, otitis media, and sepsis [13]. 
Enterobacteriaceae was the highest distributed bacteria and presented the most significant bacterial population that clinically isolated, among other Gram-negative bacteria, with more than 50\% from isolated pathogens. Enterobacteriaceae appears to be the most gram-negative bacteria with a significant distribution [14], and the incidence of infection has recently increased [15]. Enterobacteriaceae are involved in about $80 \%$ of the gram-negative bacteria responsible for urinary tract infections, sepsis, meningitis, diarrhoea, and other infections [5]. Within the Enterobacteriaceae, the E. coli was isolated from about $30 \%$ of clinical samples that were analysed on the current study. E. coli is a significant global public health problem, and it is a widely investigated bacteria and linked with many infections with a high ability to resist antimicrobial treatment [16-18]. The top dominate of $E$. coli among the Enterobacteriaceae may be due to their high genotypical diversity [19].Within the family of Enterobacteriaceae, Enterobacter spp. had been known as an important organism; these bacteria have increasingly resisted antibiotics treatment [15] and commonly cause hospital-acquired pneumonia [20]. $P$. aeruginosa was also shown to be connected with high morbidity and patients deaths therefore the bacteria is a global threat and needs complicated treatment. This pathogen can resist antimicrobial treatments and is the common infected bacteria among patients [21, 22]. The high prevalence of $P$. aeruginosa in this region could be attributed to the fact that a high antibiotic resistance rate of $P$. aeruginosa to the first line anti-pseudomonal drugs was observed in a previous study achieved in Duhok [23].

The current results revealed that the highest rate of pathogenic bacteria was observed in August, followed by October. This was comparable to various studies that showed seasonal changes in the microbial communities during different times of the year. In one study, there are significant differences in microbial composition during different seasons, with pronounced differences in summer and winter [24]. Furthermore, other reviews established that microbial activity was higher in the summer compared to winter [25]. Different factors play a role in the transmission and distribution of the microorganism in different seasons. These factors include, for example, the temperature and humidity conditions, rain, winds [24].

The percentage of pathogenic bacteria was more abundant in the female population than males. This result was in agreement with various studies indicating that women acquire bacteria in higher proportions than men $[18,26]$. There are different biological, behavioural, and social factors making the women more likely than men to be infected with pathogens [27].

To conclude, the incidence of gram-positive $S$. aureus and the gram-negative bacteria the $E$. coli infection in Duhok, Iraq, is high. There is seasonal variability in pathogen incidence with the highest rates in August. Females were more susceptible to pathogenic bacteria in comparison to males. Further investigation, a larger sample of the population, longer period with adequate surveillance programs, and active screening are required to prevent, control, and to reduce the incidence of the pathogenic bacteria.

\section{Acknowledgement}

The authors are grateful to Duhok Teaching Hospital and all microbiology laboratory staff members for allowing them to collect samples and data to complete the project. 


\section{References}

[1] Beirn, L.A., B.B. Clarke, and J.A. Crouch: Influence of host and geographic locale on the distribution of Colletotrichum cereale lineages. PLoS One, 9(5): p. e97706 (2014).

[2] Smith, K.F. and J.F. Guegan: Changing Geographic Distributions of Human Pathogens. Annu. Rev. Ecol. Evol. Syst, 41: p. 231-50 (2010).

[3] Wilhelm, S.W., G.R. LeCleir, G.S. Bullerjahn, R.M. McKay, M.A. Saxton, M.R. Twiss, and R.A. Bourbonniere: Seasonal changes in microbial community structure and activity imply winter production is linked to summer hypoxia in a large lake. FEMS Microbiol Ecol, 87(2): p. 475-85 (2014).

[4] Dufour, B. and S. La Vieille: Epidemiological surveillance of infectious diseases in France. Veterinary research, 31(2): p. 169-85 (2000).

[5] Oliveira, J. and W.C. Reygaert: Gram Negative Bacteria, in StatPearls: Treasure Island (FL) (2019)

[6] Monaco, M., F. Pimentel de Araujo, M. Cruciani, E.M. Coccia, and A. Pantosti: Worldwide Epidemiology and Antibiotic Resistance of Staphylococcus aureus. Current topics in microbiology and immunology, 409: p. 21-56 (2017).

[7] Hussein, N.R., A. Alyas, M. Majeed, and M.S. Assafi: Prevalence rate and prevalent genotypes of CA-MRSA in Kurdistan region: First report from Iraq. Int. J. Pure Appl. Sci. Technol., 27(1): p. 44-9 (2015).

[8] Assafi, M.S., R.Q. Mohammed, and N.R. Hussein: Nasal carriage rates of Staphylococcus aureus and ca-methicillin resistant Staphylococcus aureus among university students. Int. J. Microbiol. Res., 5(4): p. 123-7 (2015).

[9] Habeeb, A., N. Hussein, M. Assafi, and S. Al-Dabbagh: Methicillin resistant Staphylococcus aureus nasal colonization among secondary school students at Duhok City-Iraq. Journal of Microbiology and Infectious Diseases, 4: p. 5 (2014).

[10] Hussein, N.R., M.S. Assafi, and T. Ijaz: Methicillin-resistant Staphylococcus aureus nasal colonisation amongst healthcare workers in Kurdistan Region, Iraq. Journal of global antimicrobial resistance, 9: p. 78-81 (2017).

[11] Imohl, M., C. Fitzner, S. Perniciaro, and M. van der Linden: Epidemiology and distribution of 10 superantigens among invasive Streptococcus pyogenes disease in Germany from 2009 to 2014. PLoS One, 12(7): p. e0180757 (2017).

[12] Medina, E., Q. Goldmann, A.W. Toppel, and G.S. Chhatwal: Survival of Streptococcus pyogenes within Host Phagocytic Cells: A Pathogenic Mechanism for Persistence and Systemic Invasion. The Journal of Infectious Diseases, 187(4): p. 597-603, (2003).

[13] Cherazard, R., M. Epstein, T.L. Doan, T. Salim, S. Bharti, and M.A. Smith: Antimicrobial Resistant Streptococcus pneumoniae: Prevalence, Mechanisms, and Clinical Implications. American journal of therapeutics, 24(3): p. e361-e369 (2017).

[14] Li, X.Z., P. Plesiat, and H. Nikaido: The challenge of efflux-mediated antibiotic resistance in Gram-negative bacteria. Clinical microbiology reviews, 28(2): p. 337-418 (2015).

[15] Sanders, W.E., Jr. and C.C. Sanders: Enterobacter spp.: pathogens poised to flourish at the turn of the century. Clinical microbiology reviews, 10(2): p. 220-41 (1997).

[16] Vila, J., E. Saez-Lopez, J.R. Johnson, U. Romling, U. Dobrindt, R. Canton, C.G. Giske, et al.: Escherichia coli: an old friend with new tidings. FEMS microbiology reviews, 40(4): p. 437-463 (2016).

[17] Assafi, M.S., N.M.R. Ibrahim, N.R. Hussein, A.A. Taha, and A.A. Balatay: Urinary Bacterial Profile and Antibiotic Susceptibility Pattern among Patients with Urinary 
Tract Infection in Duhok City, Kurdistan Region, Iraq. Int. J. Pure Appl. Sci. Technol., 30(2): p. 54-63 (2015).

[18] Polse, R.F., S.Y. Yousif, and M.S. Assafi: Prevalence and antimicrobial susceptibility patterns of uropathogenic E. coli among people in Zakho, Iraq. Int. J. Res. Med. Sci., 4(4): p. 1219-1223 (2016).

[19] Lyautey, E., Z. Lu, D.R. Lapen, G. Wilkes, A. Scott, T. Berkers, T.A. Edge, and E. Topp: Distribution and Diversity of $<\mathrm{em}>$ Escherichia coli</em> Populations in the South Nation River Drainage Basin, Eastern Ontario, Canada. Applied and Environmental Microbiology, 76(5): p. 1486-1496 (2010).

[20] Holsen, M.R., L.C. Wardlow, J.A. Bazan, L.A. Fussner, K.E. Coe, and J.L. Elefritz: Clinical outcomes following treatment of Enterobacter species pneumonia with piperacillin/tazobactam compared to cefepime or ertapenem. International journal of antimicrobial agents, (2019).

[21] Ding, C., Z. Yang, J. Wang, X. Liu, Y. Cao, Y. Pan, L. Han, and S. Zhan: Prevalence of Pseudomonas aeruginosa and antimicrobial-resistant Pseudomonas aeruginosa in patients with pneumonia in mainland China: a systematic review and meta-analysis. International Journal of infectious diseases : IJID: official publication of the International Society for Infectious Diseases, 49: p. 119-28 (2016).

[22] Oliver, A., X. Mulet, C. Lopez-Causape, and C. Juan: The increasing threat of Pseudomonas aeruginosa high-risk clones. Drug resistance updates: reviews and commentaries in antimicrobial and anticancer chemotherapy, 21-22: p. 41-59 (2015).

[23] Al-Derzi, N.A.: Pattern of Resistance to Pseudomonas infection in the North of Iraq: Emphasis on the Potential Role of a Combination Antibiogram. IRAQI JOURNAL OF COMMUNITY MEDICINE, 25(2): p. 130-135 (2012).

[24] Els, N., C. Larose, K. Baumann-Stanzer, R. Tignat-Perrier, C. Keuschnig, T.M. Vogel, and B. Sattler: Microbial composition in seasonal time series of free tropospheric air and precipitation reveals community separation. Aerobiologia, 35(4): p. 671-701 (2019).

[25] Wilhelm, S.W., G.R. LeCleir, G.S. Bullerjahn, R.M. McKay, M.A. Saxton, M.R. Twiss, and R.A. Bourbonniere: Seasonal changes in microbial community structure and activity imply winter production is linked to summer hypoxia in a large lake. FEMS microbiology ecology, 87(2): p. 475-485 (2014).

[26] Fierer, N., M. Hamady, C.L. Lauber, and R. Knight: The influence of sex, handedness, and washing on the diversity of hand surface bacteria. Proceedings of the National Academy of Sciences, 105(46): p. 17994-17999 (2008).

[27] Rogers, R.G., B.G. Everett, J.M. Saint Onge, and P.M. Krueger: Social, behavioural, and biological factors, and sex differences in mortality. Demography, 47(3): p. 555-578 (2010) 